\title{
Microarray analysis identification of key pathways and interaction network of differential gene expressions during osteogenic differentiation
}

Fatemeh Khodabandehloo', Sara Taleahmad ${ }^{2}$, Reza Aflatoonian ${ }^{3}$, Farzad Rajaei ${ }^{4}$, Zahra Zandieh ${ }^{5}$, Marjan Nassiri-Asl ${ }^{4^{*}}$ (D) and Mohamadreza Baghaban Eslaminejad ${ }^{2^{*}}$

\begin{abstract}
Background: Adult bone marrow-derived mesenchymal stem cells (BM-MSCs) are multipotent stem cells that can differentiate into three lineages. They are suitable sources for cell-based therapy and regenerative medicine applications. This study aims to evaluate the hub genes and key pathways of differentially expressed genes (DEGs) related to osteogenesis by bioinformatics analysis in three different days. The DEGs were derived from the three different days compared with day 0.

Results: Gene expression profiles of GSE37558 were obtained from the Gene Expression Omnibus (GEO) database. A total of 4076 DEGs were acquired on days 8, 12, and 25. Gene ontology (GO) enrichment analysis showed that the non-canonical Wnt signaling pathway and lipopolysaccharide (LPS)-mediated signaling pathway were commonly upregulated DEGs for all 3 days. KEGG pathway analysis indicated that the PI3K-Akt and focal adhesion were also commonly upregulated DEGs for all 3 days. Ten hub genes were identified by CytoHubba on days 8, 12, and 25. Then, we focused on the association of these hub genes with the Wnt pathways that had been enriched from the protein-protein interaction (PPI) by the Cytoscape plugin MCODE.

Conclusions: These findings suggested further insights into the roles of the PI3K/AKT and Wnt pathways and their association with osteogenesis. In addition, the stem cell microenvironment via growth factors, extracellular matrix (ECM), IGF1, IGF2, LPS, and Wnt most likely affect osteogenesis by PI3K/AKT.
\end{abstract}

Keywords: Bone mesenchymal stem cells, Bioinformatics analysis, Osteogenic differentiation, Protein-protein network

\footnotetext{
* Correspondence: mnassiriasl@qums.ac.ir; eslami@royaninstitute.org

Fathemeh Khodabandehloo and Sara Taleahmad have equally contributed to the article

${ }^{4}$ Cellular and Molecular Research Center, Research Institute for Prevention of Non-Communicable Disease, Qazvin University of Medical Sciences, Qazvin,

Iran

${ }^{2}$ Department of Stem Cells and Developmental Biology, Cell Science

Research Center, Royan Institute for Stem Cell Biology and Technology,

ACECR, Tehran, Iran

Full list of author information is available at the end of the article
}

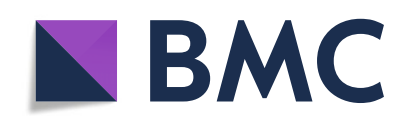

(- The Author(s). 2020 Open Access This article is licensed under a Creative Commons Attribution 4.0 International License, which permits use, sharing, adaptation, distribution and reproduction in any medium or format, as long as you give appropriate credit to the original author(s) and the source, provide a link to the Creative Commons licence, and indicate if changes were made. The images or other third party material in this article are included in the article's Creative Commons licence, unless indicated otherwise in a credit line to the material. If material is not included in the article's Creative Commons licence and your intended use is not permitted by statutory regulation or exceeds the permitted use, you will need to obtain permission directly from the copyright holder. To view a copy of this licence, visit http://creativecommons.org/licenses/by/4.0/. The Creative Commons Public Domain Dedication waiver (http://creativecommons.org/publicdomain/zero/1.0/) applies to the data made available in this article, unless otherwise stated in a credit line to the data. 


\section{Introduction}

Mesenchymal stem cells (MSCs) are multipotent and nonhematopoietic stromal cells that have the ability for self-renewal [1]. MSCs are isolated from different sources and their characteristics depend on the source $[2,3]$ from which they are obtained. It is necessary to identify the molecular mechanisms for osteogenic differentiation in bone marrow MSCs (BM-MSCs) [4] that have a high potential for osteogenesis. Because of their ability to differentiate into osteoblasts, these cells have been extensively used for regenerative medicine and the cure of bone disorders. Osteogenic differentiation is a programmed process, and the most current knowledge has been obtained by determining the role of genes such as runt-related transcription factors 2 (Runx2), distalless homeobox 5 (Dlx5), osteocalcin (OCN), and osterix (Osx) [5]. In addition, other factors involved in osteogenic differentiation include bone morphogenetic proteins (BMPs) [6], fibroblast growth factor (FGF) [7], transforming growth factor- $\beta$ (TGF- $\beta$ ) [6], hedgehog $(\mathrm{HH})$ [8], and microRNAs [9]. However, the role of many genes and the relationship between them during osteogenesis of MSCs is not completely understood, and there is a need to focus on them to improve the knowledge of tissue and bone engineering.

For this reason, several hypotheses have been considered in this study as follows: the first hypothesis is that $\mathrm{NF}-\mathrm{kB}$ is an important mediator in osteogenesis promotion by toll-like receptor 4 via the BMP2 pathway. Since TLR4 from the lipopolysaccharide (LPS)-mediated pathway plays an important role in osteogenesis. The TLR4 promotes osteogenic differentiation upon stimulation by its ligand LPS [10]. Stimulation of TLR4 by LPS in human dental pulp stem cells (hDPSCs) activated NF-kB by regulating the PI3K/AKT signaling [11]. However, the mechanisms by which NF- $\mathrm{B}$ regulates differentiation of MSCs into osteoblasts are not well known.

The second hypothesis is that the genes in focal adhesion signaling promote osteogenic differentiation, since the extracellular matrix (ECM) can affect osteogenic differentiation via focal adhesion kinase (FAK). Upon binding of the ligand to integrins, FAK becomes activated [12]. Activation of FAK is essential for MSCs to commit to osteoblasts [13]. FAK phosphorylates PI3K and mitogen-activated protein kinasesextracellular signal-regulated kinase-1/2 (MAPK-ERK1/2).

The third hypothesis is that stem-cell niche and microenvironment mediate osteogenesis through the PI3K/AKT signaling pathway, since it seems that the $\mathrm{PI} 3 \mathrm{~K} / \mathrm{AKT}$ is the central pathway in differentiating the MSCs into the osteoblast.

Furthermore, as the role of the Wnt pathway in the differentiation of MSCs into osteoblasts [14, 15], the fourth hypothesis is that both canonical and noncanonical Wnt pathways contribute to the regulation of osteogenesis. Two known signaling pathways exist for Wnt, canonical (Wnt/ $\beta$-catenin) and non-canonical. The non-canonical pathway contains the Wnt/planar cell polarity and the Wnt/calcium pathways $[16,17]$. Therefore, we examined how genes associated with the noncanonical Wnt pathway might influence the differentiation of MSCs into osteoblast. It seems that both the FZD4 and SFRP1 perform their role via both focal and non-focal pathways in osteogenesis.

To test these four hypotheses, we evaluated the gene expression profiling microarray data (GSE37558) at different time points (days 8,12, and 25) during osteogenesis of MSC and focused on the main biological processes and KEGG pathway enrichment of the differentially expressed genes (DEGs) which were derived from the three different days compared with day 0 . The highlighted pathways in our study were including LPS-mediated signaling pathway, focal adhesion, PI3K/AKT, and Wnt pathway which play important regulatory functions during the osteogenesis of hMSCs.

\section{Results \\ Identification of differentially expressed genes (DEGs)}

Analysis of microarray data from the GSE37558 study enabled us to identify 4076 total DEGs by GEO2R. There were 1234 upregulated DEGs and 1265 DEGs significantly downregulated based on the criteria of the adjusted $P$ values of $<0.01$ and $|\log \mathrm{FC}|>0.5$ (Fig. 1a, b). On day 8, there were 817 upregulated DEGs, 775 upregulated DEGs for day 12 and 1054 for day 25. There were 805 downregulated DEGs on day 8, 899 on day 12, and 1080 on day 25 . Interestingly, there were 582 DEGs with a maximum similarity that were upregulated and 629 DEGs that had maximum similarity and were downregulated on all 3 days. On day 25, a total of 267 upregulated DEGs showed the most change in expression and 228 downregulated DEGs had the most change in expression (Fig. 1a, b). Therefore, we focused on biological processes (BP) terms and pathways related to these DEGs.

\section{Gene ontology (GO) term enrichment analysis}

GO biological processes (BP) analysis showed that angiogenesis, the non-canonical Wnt signaling pathway, and lipopolysaccharide (LPS)-mediated signaling pathway were commonly upregulated DEGs on days 8, 12, and 25 (Fig. 1a). DNA replication initiation and mitotic cytokinesis were the most common downregulated DEGs for all 3 days (Fig. 1b). The GO terms including molecular function (MF) and cellular component (CC) are also shown in Supplementary Fig 1.

\section{KEGG pathway analysis}

According to KEGG pathway analysis, the PI3K-Akt signaling pathway, and focal adhesion were the most commonly upregulated DEGs for days 8, 12, and 25 (Fig. 1a) DNA replication and the cell cycle were the most 

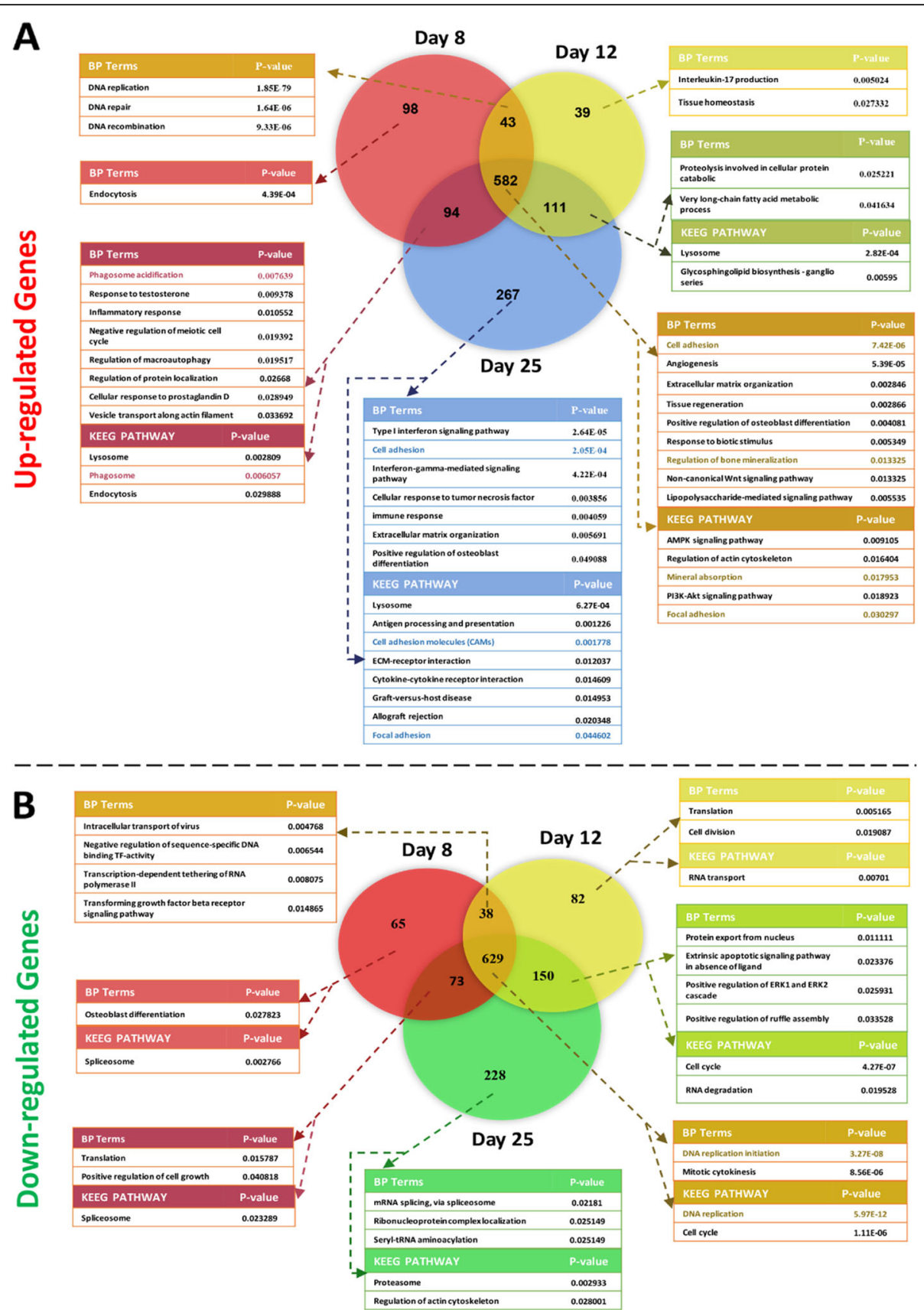

Fig. 1 Venn diagram of biological process (BP) and KEGG pathway enrichment analysis on days 8, 12, and 25. a Upregulated differentially expressed genes (DEG). b Downregulated DEGs. Common biology terms between the biological process and KEGG pathway were shown in the same color

commonly downregulated DEGs for all 3 days (Fig. 1b). Focal adhesion was also upregulated on day 25 (Fig. 1a).

\section{Construction of the protein-protein interaction (PPI) network and screening of modules}

We used Cytoscape software to visualize the PPI network of the DEGs for the testing days (days 8, 12, and 25). The modules were extracted using MCODE according to the number of nodes that were $>4$ and a node score of $>4$. Enrichment analyses of the BPs and the KEGG pathway of modules were performed (Supplementary Table 1). Supplementary Table 1 list the modules of DEGs related to osteogenesis from BP and KEGG pathway for days 8,12 , and 25 . Of note, the cell cycle and MAPK were enriched for all of the assessed days, whereas the Wnt pathway was enriched only on days 8 
and 25. Supplementary Table 2 provides a list of the top 10 hub proteins identified by CytoHubba from Cytoscape for each of the days. The association of the hub genes with the Wnt pathway was investigated. We focused on the CTNNB1( $\beta$-catenin) as the hub protein involved in the Wnt signaling in upregulated DEGs for days 8 and 25. CTNNB1 was shown in module 4, day 8 (Fig. 2c), and module 4, day 25 (Supplementary Fig 2).

\section{Validation of microarray data}

For verification of microarrays data by real-time PCR, we selected some candidate transcripts for real-time
PCR at days 8,12 , and 25. MAPK3 expression was higher in differentiated MSCs at day 8 compared to days 12 and 25 (Fig. 3a). TLR4 expression on day 25 remarkably was enhanced rather than day 8 (Fig. 3b). The CTNNB1 expression at 3 days increased compared to the control group but there were no significant differences between the three groups (Fig. 3c). Timedependent changes in the expression of the MAPK3, TLR4, CCNB1, and ITGA5 genes were concordant with array results (Fig. 3a, b, d, e), and upregulation of CTNNB1 and ITGAV genes also were in agreement at 3 days with our results (Fig. 3c, f).

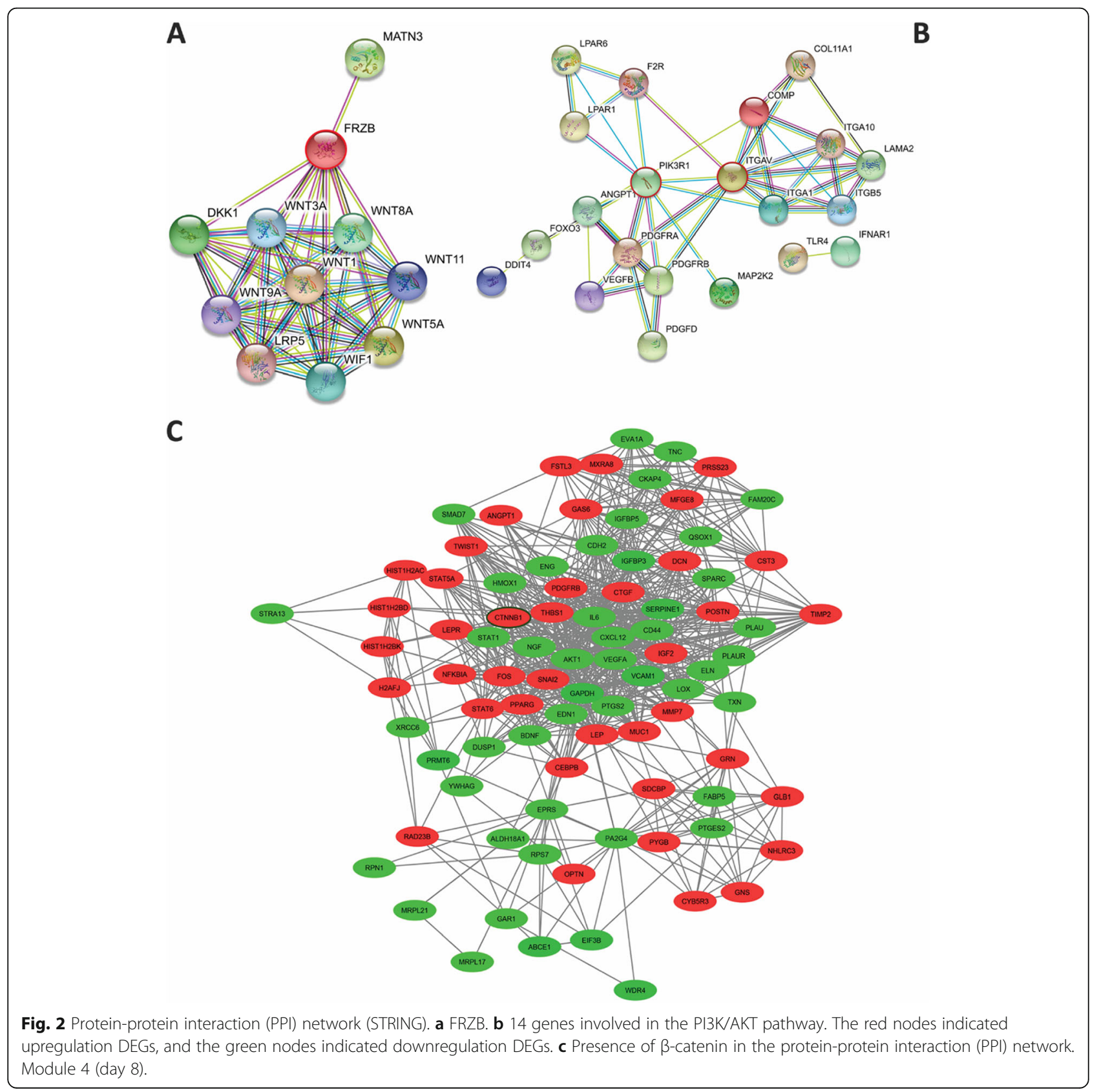



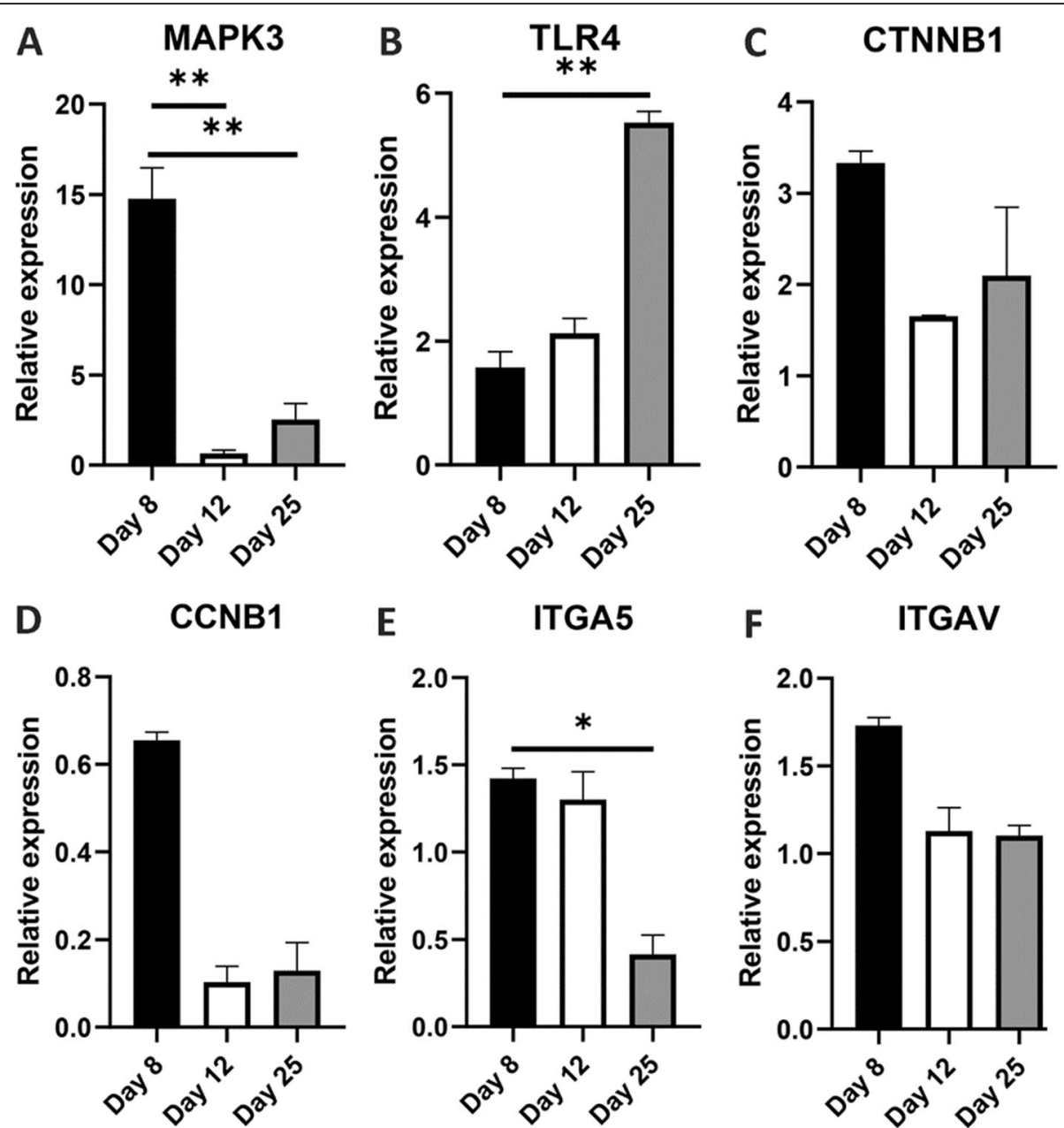

Fig. 3 Real-time PCR validation of candidate mRNA at days 8, 12, and 25. a-f the mRNA levels of MAPK3, TLR4, CTNNB1, CCNB1, ITGA5, and ITGAV were detected by real-time PCR $(n=3)$. All data are presented as mean \pm SEM. ${ }^{* *} P<0.01$

\section{Discussion}

Osteogenic differentiation is a complex process where the interaction between genes and pathways has not been fully discovered. According to the International Society of Cell Therapy protocol, MSCs should possess the following three characteristics: (i) have the capability to adhere to plastic surfaces; (ii) test positive for CD90, CD73, and CD105 and negative for CD79, CD19, CD45, CD34, CD14 or CD11b, and HLA-DR; and (iii) have the potential to differentiate into osteoblasts, chondrocytes, and adipocytes in vitro [18]. In this study, we intended to gain further insight into the identification of hub genes and key pathways during the early, middle, and late stages of osteogenic differentiation (days 0, 8, 12, and 25) as common and individual pathways.

\section{Both canonical and non-canonical Wnt pathways} contribute to regulation of osteogenesis

The Wnt pathway plays a role in the migration, growth, cell fate determination, differentiation, and $[19,20]$ bone differentiation [21]. There are four upregulated genes
(FRZB, FZD4, SFRP, and FZD1) in the non-canonical Wnt pathway. This pathway is frequently upregulated on days 8,12 , and 25 (Fig. 1a). FRZB had the highest $\log F C$ for 3 days. The overexpression of $F R Z B$ by the Wnt/ CaMKII pathway promoted osteogenic but not by activation of the canonical pathway [22]. FRZB appears to be important in the gene network (Fig. 2a). Its interaction with canonical and non-canonical ligands has been reported. FZD4 belongs to the Frizzled (FZD) family. Binding of Wnt to FZD4 activates canonical Wnt/ $\beta$-catenin signaling and promotes osteogenic differentiation. Recent studies have shown that miR-139-5p binds to CTNNB1 and FZD4, it reduces their expression and then osteogenic differentiation is decreased [23]. It was reported that mechanical stimulation promoted osteogenesis by the Wnt5a/FZD4 pathway in BM-MSCs via the non-canonical Wnt pathway [24]. SFRP1 is another upregulated gene in this pathway that has a role in the inhibition of both the canonical and non-canonical pathways [25]. 
NF-KB is an important mediator in osteogenesis promotion by toll-like receptor 4 via the BMP2 pathway LPS-mediated signaling pathway was another BP that was upregulated for all 3 days (Fig. 1a). There were five genes involved in this BP term (IL18, NFKBIA, TLR4, $S C A R B 1$, and CD14). TLR4 and CD14 from this pathway are two genes that are involved in the TLR4 signaling pathway. Three accessory proteins, including MD2, LBP, and CD14, have roles in TLR4 activation. LBP and CD14 facilitate the transfer of LPS to the TLR4/MD2 complex [26]. A study reported that TLR2 and TLR4 ligands (peptidoglycan and LPS, respectively) and TNF- $\alpha$ increase osteogenic differentiation via activation of $N F-k B$ in human adipose tissue-derived stem cells (hADSC) [27]. Taken together, these results suggest that NF- $\kappa B$ is an important transcription factor in the regulation of osteogenesis (Fig. 4a). Hess et al. have demonstrated that NF- $\mathrm{KB}$ activation which is induced by TNF- $\alpha$ promotes osteogenic differentiation by increasing BMP-2 and alkaline phosphatase (ALP) expression [28].

\section{Stem-cell niche and microenvironment mediate osteogenesis through the PI3K/AKT signaling pathway}

We observed that the PI3K/AKT pathway was upregulated in every 3 days. There were 21 genes involved in this pathway (Fig. 2b). Recently, results of a study have shown that platelet-derived growth factor (PDGF) increased osteogenic differentiation induced by TGF- $\beta$. However, PDGF alone did not affect osteogenic differentiation; rather, there was synergic cross-talk between the PI3K/AKT (PDGF mediated( and the mitogen-activated protein kinase (MAPK)/ERK kinase (TGF- $\beta$ mediated( pathways [29]. It was reported that the suppressor of PDGF promoted adipogenesis via PI3K signaling [30]. We observed that, following stimulation of growth factors $P D G F D$ and $V E G F B$, the receptor tyrosine kinases PDGFRA and PDGFRB were activated, followed by upregulation of mitogen-activated protein kinase 2 (MAP2K2) of MAPK (for more details, see the next section) and phosphoinositide-3-kinase regulatory subunit 1 (PIK3R1) that belongs to the PI3K pathway (Fig. 4a).

Although the PI3K/AKT signaling pathway is involved in the osteogenesis process in humans and mice [31, 32]. In mice, the role of this pathway is shown in endochondral ossification [33], this pathway also has a role in many MSC functions [34]. In vitro, AKT contributes to chondrogenesis and osteoblast development in metatarsal growth isolated from mice [35]. It has been reported that the activation of PI3K/AKT signaling was important in noncaveolar cholesterol-rich membrane raft likely for human MSC osteogenesis [31].

Osteogenic differentiation in rat tendon stem cells (TSCs) is affected by prostaglandin E2 that activates PI3K/ AKT signaling, resulting in osteogenic differentiation induced by BMP [36]. Interestingly, BMP-2 appears to mediate the effects of the PI3K/AKT pathway on osteogenesis. The role of BMP-2 has been shown in the commitment of progenitors into osteoblasts and in the stimulation of Runx2 and other transcription factors such as Dlx3 and Dlx5 that promote osteogenesis [37, 38]. In another study, it was suggested that AKT might not directly mediate BMP-2 expression; rather, it promotes osteogenesis possibly through a transcription factor such as the NF-kB [36]. Induction of this pathway by insulinlike growth factor (IGF) promoted osteoblast differentiation through BMP. The role of IGFs also in bone formation and development is via the PI3K/AKT pathway [32].

Mukherjee et al. have demonstrated that AKT played a role in all stages of osteogenic differentiation. The results of a study indicated that AKT2, but not AKT1, was important in osteogenic differentiation through BMP-2 that AKT2 stimulated expression of the Runx2 gene [39]. Overall, the PPI results in this pathway have revealed the interaction of the PIK3R1 and integrin subunit alpha V (ITGAV) which are important genes in this network (Fig. 2b).

\section{Upregulation of involved genes in the MAPK pathway led to osteogenesis regulation}

In our study, MAP2K2 from the PI3K-AKT pathway (on day 3) and MAPK3 (on day 8) as a hub gene were upregulated. MAP2Ks, including MEK1 (MAP2K1) and MEK2 (MAP2K2), activate ERK1 (MAPK3) and ERK2 (MAPK1) [40]. Studies have shown that the MAPK pathway is important for bone formation [41-43]. However, the role of MAPKs in osteogenesis is contradictory. A recent study has demonstrated that TRIB3 influenced proliferation and differentiation in the middle stage of differentiation by inhibiting the ERK1/2 [44]. As mentioned above, the molecular mechanism that TLR4 is involved in controlling the fate of MSCs toward osteogenesis is still uncovered. Upon MAP kinase kinases (MKKs) activation in the TLR4 pathway, p38, JNK, and ERK1/2 activated [45]. In the recent study, maximum ERK activation was shown during osteogenesis of hADSCs at day 7 when LPS stimulation was enhanced [46].

It has been recently demonstrated that JNK1 is a negative regulator of osteogenesis through BMP-2 by Runx2 phosphorylation [47]. JNK2 is needed in the late stage of osteogenic differentiation [48]. Interestingly, in one study, the results showed that JNK1 was involved in mineralization in the late stage of osteogenic differentiation and mediated increased expression of IGF2 and VEGF $\alpha$ from proangiogenic factors [49]. p38 is a positive regulator in OCN synthesis [50]. MAPK signaling could be affected by various factors such as growth factors (TGF- $\beta$, BMPs, and FGF2), integrins (ECM), and mechanical loading [51], which, in growth factors and 

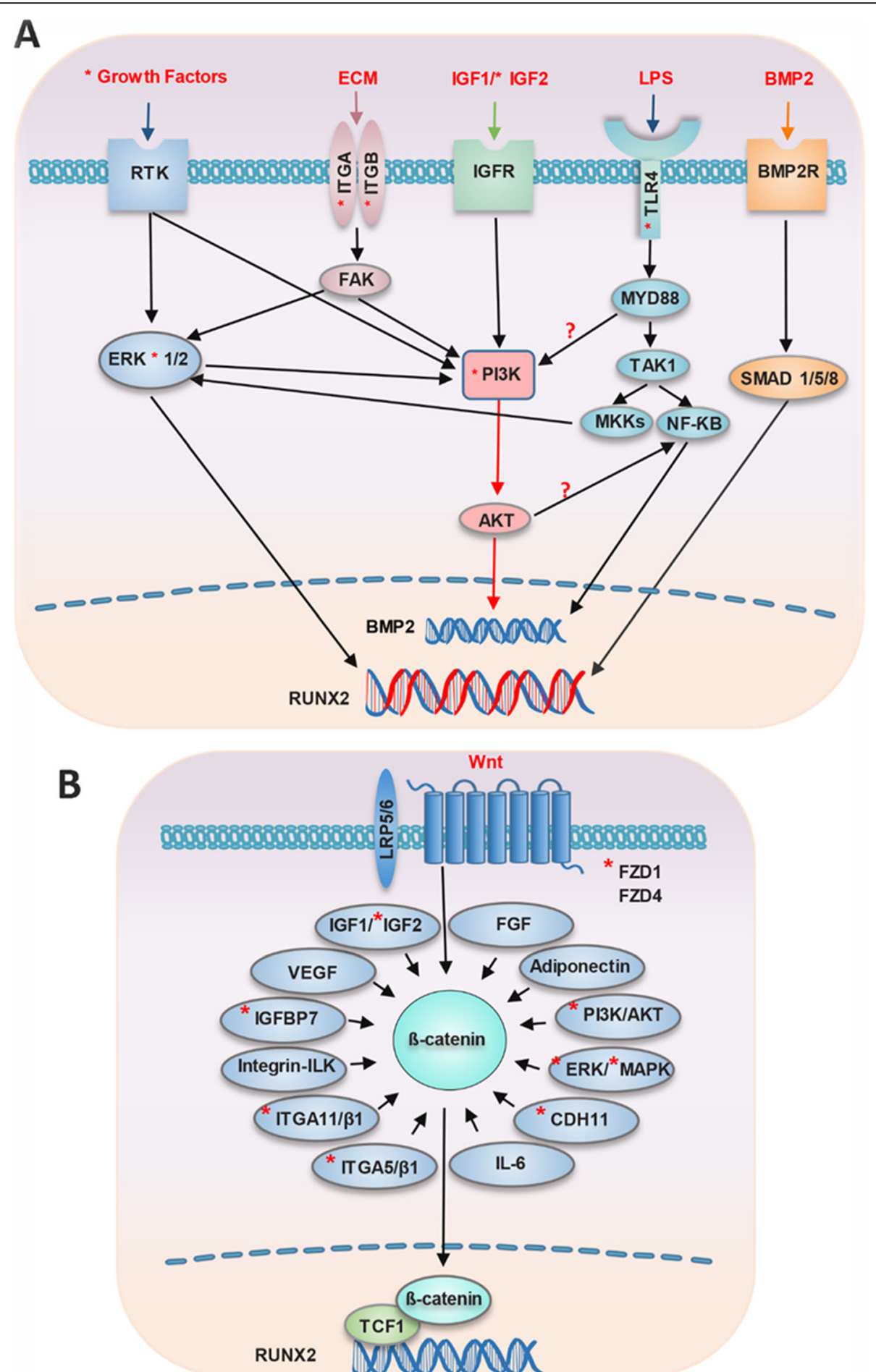

Fig. 4 Model of PI3K/AKT regulation and Wnt/B-catenin in osteogenic differentiation. Important pathways in osteoblast that promote osteogenesis via PI3K/AKT and $\beta$-catenin. a PI3K/AKT and its relationship with growth factors, ECM attachment, IGF1, IGF2, LPS, and BMP2 are illustrated in the top portion of this figure. The question mark next to PI3K/AKT/NF-KB indicates whether PI3K/AKT plays a significant role during osteogenesis directly via BMP2 signaling or indirectly through the upregulation of NF-KB. $\mathbf{b}$ The interaction or connections of genes and pathways with $\beta$-catenin are shown. Wnt/ $\beta$-catenin interacts or is affected by FGF, IGF-1/IGF-2, IGFBP7, VEGF, integrin-ILK, ITGA11/ $\beta 1$, ITGA5/ $\beta 1$, CDH1 1, ERK/MAPK, PI3K/AKT, IL-6, and adiponectin. The red star represents commonly upregulated genes on all 3 days. Only expression of MAPK3 (ERK1) at day 8, ITGA5 on days 8 and 25, and both IGFBP7 and ITGA11 at day 25 are represented. ECM, extracellular matrix; PI3K, phosphoinositide 3-kinase; MKK, MAP kinase kinases 
integrins, were consistent with our study (Fig. 4a). ERK and p38 MAP kinase could play a role in osteoblast differentiation through phosphorylation of osteogenic differentiation-related genes such as RUNX2, Osx, and DLX5 [51].

\section{Genes in focal adhesion signaling promote osteogenic differentiation}

There were 14 genes (ITGA1, ITGA10, ITGB5, CTNNB1, MYL9, VEGFB, LAMA2, COMP, ITGAV, PDGFRA, PDGFRB, PDGFD, COL11A1, and PIK3R1) involved in the focal adhesion pathway that upregulated on days 825 . We have observed the upregulation of integrin subunit alpha 5 (ITGA5) on days 8 and 12. Hamidouche et al. noted that FAK/ERK1/2-MAPKs and PI3K signaling pathways promoted osteogenic differentiation through induction of ITGA5 hMSCs [52]. It has been reported that activation of ITGA5 induced both IGF2 and IGFBP2 expressions via FAK, ERK1/2, and PI3K signaling, which resulted in osteogenic differentiation in hMSCs [53]. In another study, cilengitide (a cyclic RGD pentapeptide) is an ITGAV inhibitor [54] that can abolish ossification in BM-MSCs [55]. The interaction between osteopontin and integrin $\alpha \mathrm{v} / \beta 1$ induced osteogenesis and inhibited adipogenesis in MSCs [56]. Therefore, the binding of osteopontin, fibronectin, and other molecules involved in osteogenic differentiation could determine the possible balance between MSCs that commit toward adipogenic or osteogenic differentiation [57]. We have observed the upregulation of cadherin 11 (CDH11) on all 3 days. CDH11 is involved in cell connections and has a role in cell signaling. CDH11 is expressed in osteoblasts osteogenesis. Its role in osteoblast commitment and osteogenic differentiation has been reported [58]. Our data showed that genes related to cell adhesion such as ITGA11 and insulin-like growth factor binding protein 7 (IGFBP7) also upregulated on day 25. Integrin $\alpha 11$ (ITGA11) is a receptor for osteolectin that actives the Wnt pathway and promotes osteogenesis [59]. Recently, Zhang et al. also reported that the IGFBP7 gene promoted osteogenic differentiation of hBM-MSCs by upregulation of the $\beta$ catenin pathway [60].

\section{Relationship between hub genes and Wnt pathway during osteogenesis}

The role of important hub genes including interleukin (IL)-6, AKT1, VEGFA, CDK1, PLK1, CDC20, CCNA2, MAPK3 (on day 8), and CTNNB1 (on days 8 and 25) in the Wnt pathway was studied (Supplementary Table 2).

The inhibitory effects of IL-6 in osteoblast differentiation in rheumatoid arthritis are due to its negative interaction with the Wnt pathway [61]. In a study, Li et al. suggested that the effect of IL- 6 on inhibition of osteogenic differentiation is due to its inhibitory effect on the canonical Wnt pathway [62].

AKT1 is an important gene in the PI3K/AKT pathway. Studies have been conducted about the cross-talk between Wnt/B-catenin and PI3K/AKT signaling pathways $[63,64]$. Han et al. have reported that inhibition of PI3K/AKT suppressed transcription through $\beta$-catenin in glioblastoma cells [64]. $\beta$-catenin can be directly phosphorylated at Ser552 by AKT, which separates it from cell-cell contact and increases translocation of $\beta$ catenin into the nucleus, both in vitro and in vivo [65].

VEGFA plays a pivotal role in angiogenesis. Numerous studies have assessed the role played by VEGFA in linking osteogenesis and angiogenesis [66, 67]. In osteoblastic and endothelial cells, VEGF induced bone formation through the $\beta$-catenin pathway [68]. Inhibition of $\beta$-catenin or knockdown of Wnt4 in the MSCs led to the return of proangiogenic effects induced by Wnt signaling [69].

CDK1, PLK1, CDC20, and CCNA2 are genes involved in the cell cycle. Proliferation and differentiation have opposite connections [70]. In agreement with previous studies, the genes related to the cell cycle were downregulated [71, 72]. The association between the Wnt system and genes related to the cell cycle during osteogenesis was less observed. A study suggested that the induction of Wnt/ $\beta$-catenin, by LRP6 phosphorylation is regulated via Cyclin Y/CDK at the G2/M phase [73]. The canonical Wnt pathway also plays an important role in cell cycle control [74].

MAPK3 is related to the MAPK pathway. The results showed that the ERK interacts with the $\mathrm{Wnt} / \beta$-catenin signaling pathway. It has also been shown that the ERK pathway is involved in the differentiation of osteoblasts through the regulation of RUNX2, $\beta$-catenin, and ATF4 [43]. The Wnt pathway is indirectly impacted by ERK/ MAPK signaling via inhibition of GSK-3 $\beta$ by p38, JNK, and ERK [75].

The role of $\beta$-catenin was implicated in both canonical Wnt pathways and cell-cell adhesion $[16,76]$. In the canonical pathway, frizzled and LRP5/6 are activated by Wnt ligands. In the presence of Wnt ligands, the destruction complex (GSK3, AXIN, and APC) is inhibited and this inhibition helps the stabilization and translocation of $\beta$-catenin to the nucleus [77-80]. There is a site on promoter of Runx2 for $\beta$-catenin/TCF-1, which activates expression of this gene and promotes osteogenic differentiation [81]. Tornero-Esteban et al. investigated the involvement of the Wnt and possible compensatory mechanisms involve in the osteoarthritis (OA) pathophysiology. They showed the increased levels of $\beta$ catenin in OA-MSCs did not accompany increased osteogenic suggesting that compensatory mechanisms are involved in modulating transcriptional of osteogenic 
differentiation [82]. Due to the important role of $\beta$-catenin in the Wnt pathway, in the next section, we evaluated the $\beta$-catenin that interacts/or is affected by other pathways.

\section{The Wnt/ $\beta$-catenin pathway could interacts/or was} affected by other pathways associated with osteogenesis The interaction between integrin-related signaling molecules and the Wnt pathway suggested that integrin receptors are associated with integrin-linked kinase (ILK) [83]. GSK3 $\beta$ is phosphorylated following the activation of ILK [84]. Activation of Wnt/ $/$-catenin and PI3K-Akt signaling pathways drive osteogenic differentiation upon primed $\alpha 5 \beta 1$ integrin using peptides in mesenchymal skeletal cells [85]. Another study showed that osteolectin/ $\alpha 11 \beta 1$ results in Wnt pathway activation that increased nuclear $\beta$-catenin and finally promoted osteogenesis [59]. CDH11 is involved in osteoblast committed into the osteogenic lineage. Interestingly, adipogenesis was not affected by CDH11 and it may be mediated via $\beta$-catenin [58]. A relationship between growth factors and the Wnt signaling pathway during osteogenesis has been reported. (IGF)-I and IGF-II can also affect the $\beta$-catenin signaling pathway $[86,87]$. IGFBP7 also induced osteogenesis at day 25. FGF interacts with the $\mathrm{Wnt} / \beta$-catenin pathway in osteogenesis during the regulation of the transcription factor of Osx [88]. The role of adiponectin as an adipocytokine has been shown in bone formation through the $\mathrm{Wnt} / \beta$-catenin pathway [89]. Overall, Wnt/ $\beta$-catenin interacts or is affected by PI3K/AKT, ERK/MAPK, CDH11, integrins (integrin-ILK, integrin $\alpha 5 \beta 1$, integrin $\alpha 11 \beta 1$ ), growth factors (FGF, IGF1/ IGF2, IGFBP7, and VEGF), IL6, and adiponectin (Fig. 4b).

\section{Conclusion}

In summary, PI3K and Wnt signaling are important pathways in osteogenic differentiation. IGF2 links integrin, PI3K/AKT, and MAPK (JNK1). The extracellular environment can affect osteogenic differentiation PI3K/AKT mediated via the following: (1) LPS by TLR4; (2) integrins from ECM by the binding of substances such as osteolectin and osteocalcin, and activation of the FAK/MAPK pathway; (3) IGF1 and IGF2 through IGFRs; (4) growth factors via RTK; and (5) beta-catenin via Wnt pathway. Whether PI3K/AKT promotes osteogenesis through BMP2 directly or via NF-kB should be investigated. Taken together, this study provides further insight into the role of signaling pathways and their interaction in determining the fate of mesenchymal stem cells into osteoblast.

\section{Materials and methods}

\section{Microarray data analysis}

Raw data related to expression profiling of GSE37558 [90] were taken from the Gene Expression Omnibus (GEO, http://www.ncbi.nlm.nih.gov/geo/) database. GPL6947 platforms (Illumina HumanHT-12 v3.0 Gene Expression beadchip) were used for the gene expression profiles. In this study, we selected 13 samples from human BM-derived MSCs (hBM-MSCs) that had been cultured in osteogenic differentiation medium at four-time points (days $0,8,12$, and 25). The control group comprised four replicates from day 0 , and the differentiated groups included three replicates for each time point (days 8, 12, and 25). Sample accession (GSM) of GSE37558 related to this study in four different days are shown in Table 1.

\section{Identification of differential gene expressions (DEGs)}

GEO2R (http://www.ncbi.nlm.nih.gov/geo/geo2r/) was used to identify the DEGs between the control group (day 0 ) and differentiated groups (days 8, 12, and 25). The DEGs were identified with adjusted $P$ values of $<$ 0.01 and $|\operatorname{logFC}|>0.5$.

\section{Gene ontology (GO) and pathway enrichment analysis}

Gene ontology (GO) for biological processes (BP), molecular function (MF), and cellular component (CC) in addition to KEGG pathway enrichment analyses of differential gene expressions were obtained using the Database for Annotation Visualization and Integrated Discovery (DAVID; https://david.ncifcrf.gov) for up- and downregulated DEGs. $P$ values of less than 0.05 were considered to be the criteria. DAVID was also used to analyze both GO ontology and the KEGG pathway of the modules. Venn diagrams (http://bioinfogp.cnb.csic. es/tools/venny/index.html) were used to identify up- and downregulated DEGs for different days.

\section{Construction of protein-protein interaction (PPI) network and screening of modules}

We used the Search Tool for the Retrieval of Interacting Genes (STRING; version 11; string-db.org/) database to analyze the interactions among the DEGs. A cutoff value of greater than 0.4 was used to evaluate the proteinprotein interaction (PPI) network. Visualization of the PPI networks was performed by Cytoscape (version 3.7.1, http://www.cytoscape.org/). The Cytoscape StringApp was used to retrieve the functional enrichment of up- and downregulated DEGs at days 8, 12, and 25. The top 10 proteins were ranked by topological analysis methods using CytoHubba. We found the most similar proteins in the following methods: MNC, degree, EPC,

Table 1 List of samples accession (GSM) used at different fourtime points (days 0, 8, 12, and 25)

\begin{tabular}{ll}
\hline Time point & Sample accession \\
\hline 0 & GSM921574, GSM921575, GSM921576, and GSM921577 \\
8 & GSM921581, GSM921582, and GSM921583 \\
12 & GSM921584, GSM921585, and GSM921586 \\
25 & GSM921587, GSM921588, and GSM921589 \\
\hline
\end{tabular}


Table $\mathbf{2}$ List of primers used for real-time PCR to validate microarray analysis

\begin{tabular}{|c|c|c|c|}
\hline Target gene & Primer sequence & Accession number & Product size \\
\hline GAPDH & $\begin{array}{l}\text { F: CTCATTTCCTGGTATGACAACGA } \\
\text { R: CTTCCTCTTGTGCTCTTG }\end{array}$ & NM_001357943.2 & $122 \mathrm{bp}$ \\
\hline CTNNB1 & $\begin{array}{l}\text { F: AATGCTTGGTTCACCAGTG } \\
\text { R: GGCAGTCTGTCGTAATAGCC }\end{array}$ & NM_001330729.2 & $176 \mathrm{bp}$ \\
\hline MAPK3 & $\begin{array}{l}\text { F: TGACCATATCTGCTACTTCCTC } \\
\text { R: GGTATAGCCCTTGGAGTTCAG }\end{array}$ & NM_001040056.3 & $250 \mathrm{bp}$ \\
\hline ITGAV & $\begin{array}{l}\text { F: GCAACAGGCAATAGAGAT } \\
\text { R: TGCTGAATCCTCCTTGACAA }\end{array}$ & NM_002210.5 & $262 \mathrm{bp}$ \\
\hline ITGA5 & $\begin{array}{l}\text { F: GCTGTGACTACTTTGCCGTG } \\
\text { R: CGAGTTGTTGAGATTCTTGCTG }\end{array}$ & NM_002205.5 & $176 \mathrm{bp}$ \\
\hline CCNB1 & $\begin{array}{l}\text { F: GCTGGGTGTAGGTCCTTG } \\
\text { R: CCTGCCATGTTGATCTTCG }\end{array}$ & NM_031966.4 & $149 \mathrm{bp}$ \\
\hline TLR4 & $\begin{array}{l}\text { F: TGATGTCTGCCTCGCGCCTG } \\
\text { R: AACCACCTCCACGCAGGGCT }\end{array}$ & NM_138554.5 & $98 \mathrm{bp}$ \\
\hline
\end{tabular}

and closeness. Key modules in the PPI network were identified by using the Cytoscape plugin Molecular Complex Detection (MCODE, version 1.5.1). The criteria were both a node score and node number greater than 4.

\section{Cell culture}

Human BM-MSCs were obtained from the Royan Stem Cell Bank. The study protocol was approved by the Royan Institute ethical committee board. All the experiments were designed in three biological replicates. The cells were cultured in $\alpha$-MEM (Gibco, cat. no. 12571) with 10\%FBS (Gibco, cat. no. 10082139), $100 \mathrm{U} / \mathrm{ml}$ penicillin (Gibco, cat. no. 15070063), and $100 \mathrm{mg} / \mathrm{ml}$ streptomycin. After $24 \mathrm{~h}$, the cells were induced with osteogenic medium containing DMEM supplemented with $10 \% \mathrm{FBS}, 50 \mu \mathrm{g} / \mathrm{mL}$ ascorbic acid (Sigma-Aldrich, cat. no. A8960), $10 \mathrm{mM} \beta$-glycerophosphate (Sigma-Aldrich, cat. no. 154804-51-0), and $1 \times 10^{-8} \mathrm{M}$ dexamethasone (Sigma-Aldrich, cat. no. D4902) for days 8, 12, and 25. The media was replaced every 3 days, and the characterization of osteogenic was performed by alizarin red and Oil Red O staining (Supplementary Fig 3).

\section{Validation of microarrays data by real-time PCR}

The real-time PCR was performed for the validation of microarray data. The expression level of mRNAs for CTNNB1, MAPK3, ITGAV, ITGA5, CCNB1, and TLR4 were evaluated by a real-time PCR System. We isolated total RNA by TRI reagent (Sigma-Aldrich, T9424) from cultured MSCs in osteogenic medium on days 8,12 , and 25. cDNA was synthesized from $1 \mu \mathrm{g}$ total RNA using the PrimeScriptTMRT reagent Kit (Takara Perfect Real Time). All reactions were carried out duplicate by using StepOnePlus Real-time PCR System (Applied biosystems life technologies, ABi). GAPDH was used as a reference gene to normalize the expression of all target genes. All tested groups compared to the control group (day 0). The $\Delta \triangle$ Ct method was used to analyze Q-RT PCR data for quantitative analysis. The specific primers sequence for each gene is shown in Table 2 .

\section{Statistical analysis}

Statistical analyses were performed using Prism statistical software (version 8). The statistical significance of the differences between groups at days 8, 12, and 25 was performed by the Kruskal-Wallis test.

\section{Supplementary Information}

The online version contains supplementary material available at https://doi. org/10.1186/s40246-020-00293-1.

Additional file 1:. Supplementary Figure 1. Venn diagram of molecular function (MF) and cellular component (CC) on days 8, 12, and 25. a Upregulated differentially expressed genes (DEG). b Downregulated DEGs. Supplementary Table 2. Hub genes for up- and downregulated genes ranked in CytoHubba. Supplementary Figure 2. Protein-protein interaction (PPI) network (STRING). Supplementary Figure 3. The ability of MSCs to differentiate into osteoblast and adipocyte

Additional file 2:. Supplementary Table 1. Biological process (BP) and KEGG enrichment analyses of the differentially expressed genes in the Modules. (XLS $42 \mathrm{~kb}$ )

\section{Abbreviations}

BM-MSC: Bone marrow-derived mesenchymal stem cell; DEG: Differentially expressed gene; GO: Gene ontology; LPS: Lipopolysaccharide; KEGG: Kyoto Encyclopedia of Genes and Genomes; MCODE: Molecular Complex Detection; PPI: Protein-protein interaction; ECM: Extracellular matrix Runx2: Runt-related transcription factors 2; DIx5: Distal-less homeobox 5; OCN: Osteocalcin; Osx: Osterix; BMP: Bone morphogenetic protein; GEO: Gene Expression Omnibus; BP: Biological processes; MF: Molecular function; CC: Cellular component; DAVID: Database for Annotation Visualization and Integrated Discovery; STRING: Search Tool for the Retrieval of Interacting Genes; ALP: Alkaline phosphatase; VEGFB: Vascular endothelial growth factor B; hADSC: Human adipose tissue-derived stem cell; hDPSC: Human dental pulp stem cell; PDGF: Platelet-derived growth factor; MAP2K2: Mitogen-activated protein kinase 2; PIK3R1: Phosphoinositide-3kinase regulatory subunit 1; TSC: Tendon stem cell; MAPK: Mitogen-activated protein kinases; ERK1/2: Extracellular signal-regulated kinase-1/2;

CDH11: Cadherin 11; ILK: Integrin-linked kinase; MKK: MAP kinase kinase 


\section{Acknowledgements}

The study was supported by the Royan Institute and the Qazvin University of Medical Sciences.

\section{Authors' contributions}

FK performed the RNA isolation experiment and the bioinformatics and comparative analysis and contributed to the manuscript writing. ST performed the bioinformatics and comparative analysis and contributed to the manuscript writing. RA participated in the manuscript design and assisted in the drafting of the manuscript. ZZ and FR participated in the coordination of the study and edited the manuscript. MNA and MRB participated in its design and revised the manuscript. The authors read and approved the final manuscript.

\section{Funding}

Not applicable.

\section{Availability of data and materials}

All data used and analyzed during this study are included in this published article and its supplementary information files. The other data including realtime PCR data are available from the corresponding author on reasonable request.

\section{Ethics approval and consent to participate}

The study protocol was approved by the Royan Institute ethical committee board.

\section{Consent for publication}

Not applicable.

\section{Competing interests}

The authors declare that they have no competing interests.

\section{Author details}

'Department of Molecular Medicine, Qazvin University of Medical Sciences, Qazvin, Iran. ${ }^{2}$ Department of Stem Cells and Developmental Biology, Cell Science Research Center, Royan Institute for Stem Cell Biology and Technology, ACECR, Tehran, Iran. ${ }^{3}$ Department of Endocrinology and Female Infertility, Reproductive Biomedicine Research Center, Royan Institute for Reproductive Biomedicine, ACECR, Tehran, Iran. ${ }^{4}$ Cellular and Molecular Research Center, Research Institute for Prevention of Non-Communicable Disease, Qazvin University of Medical Sciences, Qazvin, Iran. ${ }^{5}$ Department of Anatomy, School of Medicine, Iran University of Medical Sciences, Tehran, Iran.

Received: 24 June 2020 Accepted: 13 November 2020

Published online: 25 November 2020

\section{References}

1. Qiu X, Jia B, Sun X, Hu W, Chu H, Xu S, et al. The critical role of long noncoding RNA in osteogenic differentiation of human bone marrow mesenchymal stem cells. BioMed research international. 2017;2017.

2. Zuk PA, Zhu M, Mizuno H, Huang J, Futrell JW, Katz AJ, et al. Multilineage cells from human adipose tissue: implications for cell-based therapies. Tissue engineering. 2001;7(2):211-28.

3. d'Aquino R, Graziano A, Sampaolesi M, Laino G, Pirozzi G, De Rosa A, et al. Human postnatal dental pulp cells co-differentiate into osteoblasts and endotheliocytes: a pivotal synergy leading to adult bone tissue formation. Cell death and differentiation. 2007;14(6):1162.

4. Wang L, Wang Y, Li Z, Li Z, Yu B. Differential expression of long noncoding ribonucleic acids during osteogenic differentiation of human bone marrow mesenchymal stem cells. International Orthopaedics. 2015;39(5):1013-9.

5. Kirkham G, Cartmell S. Genes and proteins involved in the regulation of osteogenesis. Topics in tissue engineering. 2007;3:1-22.

6. Hayrapetyan A, Jansen JA, van den Beucken JJ. Signaling pathways involved in osteogenesis and their application for bone regenerative medicine. Tissue Engineering Part B: Reviews. 2014;21(1):75-87.

7. Marie P. Fibroblast growth factor signaling controlling osteoblast differentiation. Gene. 2003;316:23-32.
8. Ehlen HW, Buelens LA, Vortkamp A. Hedgehog signaling in skeletal development. Birth Defects Research Part C: Embryo Today: Reviews. 2006; 78(3):267-79.

9. Wang J, Liu S, Li J, Zhao S, Yi Z. Roles for miRNAs in osteogenic differentiation of bone marrow mesenchymal stem cells. Stem cell research \& therapy. 2019;10(1):197.

10. He X, Wang H, Jin T, Xu Y, Mei L, Yang J. TLR4 activation promotes bone marrow MSC proliferation and osteogenic differentiation via Wnt3a and Wnt5a signaling. PLoS One. 2016;11(3):e0149876.

11. He W, Wang Z, Zhou Z, Zhang Y, Zhu Q, Wei K, et al. Lipopolysaccharide enhances Wnt5a expression through toll-like receptor 4, myeloid differentiating factor 88, phosphatidylinositol 3-OH kinase/AKT and nuclear factor kappa B pathways in human dental pulp stem cells. Journal of endodontics. 2014;40(1):69-75.

12. Guan J-L. Focal adhesion kinase in integrin signaling. Matrix Biology. 1997; 16(4):195-200.

13. Salasznyk RM, Klees RF, Williams WA, Boskey A, Plopper GE. Focal adhesion kinase signaling pathways regulate the osteogenic differentiation of human mesenchymal stem cells. Experimental cell research. 2007;313(1):22-37.

14. Thorfve A, Svala E, Brisby H, Thomsen P, Lindahl A. Madindoline A affects the osteogenic potential and the Wnt signaling pathways during osteogenic differentiation of human mesenchymal stem cells in vitro. J Bone Marrow Res. 2014;2(151):2.

15. Cao Y, Yang H, Jin L, Du J, Fan Z. Genome-wide DNA methylation analysis during osteogenic differentiation of human bone marrow mesenchymal stem cells. Stem cells international. 2018;2018.

16. Komiya Y, Habas R. Wnt signal transduction pathways. Organogenesis. 2008; 4(2):68-75.

17. Pataki CA, Couchman JR, Brábek J. Wnt signaling cascades and the roles of syndecan proteoglycans. Journal of Histochemistry \& Cytochemistry. 2015; 63(7):465-80.

18. Dominici M, Le Blanc K, Mueller I, Slaper-Cortenbach I, Marini F, Krause D, et al. Minimal criteria for defining multipotent mesenchymal stromal cells. The International Society for Cellular Therapy position statement. Cytotherapy. 2006;8(4):315-7.

19. Moon RT, Shah K. Signalling polarity. Nature. 2002;417(6886):239-40.

20. Golestaneh N, Beauchamp E, Fallen S, Kokkinaki M, Uren A, Dym M. Wnt signaling promotes proliferation and stemness regulation of spermatogonial stem/progenitor cells. Reproduction. 2009;138(1):151.

21. Houschyar KS, Tapking C, Borrelli MR, Popp D, Duscher D, Maan ZN, et al. Wnt pathway in bone repair and regeneration-what do we know so far. Frontiers in cell and developmental biology. 2019;6:170.

22. Thysen S, Cailotto F, Lories R. Osteogenesis induced by frizzled-related protein (FRZB) is linked to the netrin-like domain. Laboratory Investigation. 2016;96(5):570.

23. Long H, Sun B, Cheng L, Zhao S, Zhu Y, Zhao R, et al. miR-139-5p represses BMSC osteogenesis via targeting Wnt/ $\beta$-catenin signaling pathway. DNA and cell biology. 2017;36(8):715-24.

24. Gu Q, Tian H, Zhang K, Chen D, Chen D, Wang X, et al. Wnt5a/FZD4 mediates the mechanical stretch-induced osteogenic differentiation of bone mesenchymal stem cells. Cellular Physiology and Biochemistry. 2018;48(1):215-26.

25. Yao W, Cheng Z, Shahnazari M, Dai W, Johnson ML, Lane NE. Overexpression of secreted frizzled-related protein 1 inhibits bone formation and attenuates parathyroid hormone bone anabolic effects. Journal of Bone and Mineral Research. 2010;25(2):190-9.

26. Kim SJ, Kim HM. Dynamic lipopolysaccharide transfer cascade to TLR4/MD2 complex via LBP and CD14. BMB reports. 2017;50(2):55.

27. Cho HH, Shin KK, Kim YJ, Song JS, Kim JM, Bae YC, et al. NF-kB activation stimulates osteogenic differentiation of mesenchymal stem cells derived from human adipose tissue by increasing TAZ expression. Journal of cellular physiology. 2010;223(1):168-77.

28. Hess K, Ushmorov A, Fiedler J, Brenner RE, Wirth T. TNFa promotes osteogenic differentiation of human mesenchymal stem cells by triggering the NF-KB signaling pathway. Bone. 2009;45(2):367-76.

29. Yokota J, Chosa N, Sawada S, Okubo N, Takahashi N, Hasegawa T, et al. PDGF-induced PI3K-mediated signaling enhances the TGF- $\beta$-induced osteogenic differentiation of human mesenchymal stem cells in a TGF- $\beta$ activated MEK-dependent manner. International journal of molecular medicine. 2014;33(3):534-42. 
30. Fitter S, Vandyke K, Gronthos S, Zannettino AC. Suppression of PDGFinduced PI3 kinase activity by imatinib promotes adipogenesis and adiponectin secretion. Journal of molecular endocrinology. 2012;48(3): 229-40.

31. Baker N, Sohn J, Tuan RSJScr, therapy. Promotion of human mesenchymal stem cell osteogenesis by PI3-kinase/Akt signaling, and the influence of caveolin-1/cholesterol homeostasis. 2015;6(1):238.

32. Ghosh-Choudhury N, Abboud SL, Nishimura R, Celeste A, Mahimainathan L, Choudhury GG. Requirement of BMP-2-induced phosphatidylinositol 3kinase and Akt serine/threonine kinase in osteoblast differentiation and Smad-dependent BMP-2 gene transcription. Journal of Biological Chemistry. 2002;277(36):33361-8.

33. Ulici V, Hoenselaar KD, Gillespie JR, Beier FJBdb. The PI3K pathway regulates endochondral bone growth through control of hypertrophic chondrocyte differentiation. 2008:8(1):40

34. Chen J, Crawford R, Chen C, Xiao YJTEPBR. The key regulatory roles of the PI3K/Akt signaling pathway in the functionalities of mesenchymal stem cells and applications in tissue regeneration. 2013;19(6):516-28.

35. Mukherjee A, Rotwein P. Akt promotes BMP2-mediated osteoblast differentiation and bone development. J Cell Sci. 2009;122(5):716-26.

36. Liu J, Chen L, Tao X, Tang KJB, communications br. Phosphoinositide 3kinase/Akt signaling is essential for prostaglandin E2-induced osteogenic differentiation of rat tendon stem cells. 2013;435(4):514-9.

37. Hassan MQ, Javed A, Morasso MI, Karlin J, Montecino M, Van Wijnen AJ, et al. Dlx3 transcriptional regulation of osteoblast differentiation: tempora recruitment of Msx2, Dlx3, and Dlx5 homeodomain proteins to chromatin of the osteocalcin gene. 2004;24(20):9248-61.

38. Lee M-H, Kim Y-J, Yoon W-J, Kim J-I, Kim B-G, Hwang Y-S, et al. Dlx5 specifically regulates Runx2 type II expression by binding to homeodomain-response elements in the Runx2 distal promoter. 2005: 280(42):35579-87.

39. Mukherjee A, Wilson EM, Rotwein P. Selective signaling by Akt2 promotes bone morphogenetic protein 2-mediated osteoblast differentiation. Molecular and cellular biology. 2010;30(4):1018-27.

40. Greenblatt MB, Shim J-H, Glimcher LH. Mitogen-activated protein kinase pathways in osteoblasts. Annual review of cell and developmental biology. 2013:29:63-79.

41. Ge C, Xiao G, Jiang D, Franceschi RT. Critical role of the extracellular signalregulated kinase-MAPK pathway in osteoblast differentiation and skeletal development. The Journal of cell biology. 2007;176(5):709-18.

42. Greenblatt MB, Shim J-H, Zou W, Sitara D, Schweitzer M, Hu D, et al. The p38 MAPK pathway is essential for skeletogenesis and bone homeostasis in mice. The Journal of clinical investigation. 2010;120(7):2457-73.

43. Kim J-M, Yang Y-S, Park KH, Oh H, Greenblatt MB, Shim J-H. The ERK MAPK pathway is essential for skeletal development and homeostasis. International journal of molecular sciences. 2019;20(8):1803.

44. Zhang C, Hong F-F, Wang C-C, Li L, Chen J-L, Liu F, et al. TRIB3 inhibits proliferation and promotes osteogenesis in hBMSCs by regulating the ERK1/ 2 signaling pathway. Scientific reports. 2017;7(1):10342.

45. Barton GM. Medzhitov RJS. Toll-like receptor signaling pathways. 2003; 300(5625):1524-5.

46. Hwa Cho H, Bae YC. Jung JSJSc. Role of toll-like receptors on human adipose-derived stromal cells. 2006;24(12):2744-52.

47. Huang YF, Lin JJ, Lin CH, Su Y, Hung SC. C-Jun N-terminal kinase 1 negatively regulates osteoblastic differentiation induced by BMP2 via phosphorylation of Runx2 at Ser104. Journal of Bone and Mineral Research. 2012;27(5):1093-105.

48. Matsuguchi T, Chiba N, Bandow K, Kakimoto K, Masuda A, Ohnishi T. JNK activity is essential for Atf4 expression and late-stage osteoblast differentiation. Journal of Bone and Mineral Research. 2009;24(3):398-410.

49. Xu R, Zhang C, Shin DY, Kim JM, Lalani S, Li N, et al. C-Jun N-terminal kinases (JNKS) are critical mediators of osteoblast activity in vivo. Journal of Bone and Mineral Research. 2017;32(9):1811-5

50. Kozawa O, Hatakeyama D, Uematsu T. Divergent regulation by p44/p42 MAP kinase and p38 MAP kinase of bone morphogenetic protein-4stimulated osteocalcin synthesis in osteoblasts. Journal of cellular biochemistry. 2002;84(3):583-9.

51. Franceschi RT, Ge C. Control of the osteoblast lineage by mitogen-activated protein kinase signaling. Current molecular biology reports. 2017;3(2):122-32.

52. Hamidouche $Z$, Fromigué $O$, Ringe J, Häupl $T$, Vaudin $P$, Pagès J-C, et al. Priming integrin a5 promotes human mesenchymal stromal cell osteoblast differentiation and osteogenesis. Proceedings of the National Academy of Sciences. 2009;106(44):18587-91.

53. Hamidouche Z, Fromigué O, Ringe J, Häupl T, Marie PJ. Crosstalks between integrin alpha 5 and IGF2/IGFBP2 signalling trigger human bone marrowderived mesenchymal stromal osteogenic differentiation. BMC cell biology. 2010;11(1):44.

54. Tucker GC. Integrins: molecular targets in cancer therapy. Current oncology reports. 2006;8(2):96-103.

55. Logan NJ, Camman M, Williams G, Higgins CA. Demethylation of ITGAV accelerates osteogenic differentiation in a blast-induced heterotopic ossification in vitro cell culture model. Bone. 2018;117:149-60.

56. Chen Q, Shou P, Zhang L, Xu C, Zheng C, Han Y, et al. An osteopontinintegrin interaction plays a critical role in directing adipogenesis and osteogenesis by mesenchymal stem cells. Stem cells. 2014;32(2):327-37.

57. Ramazzotti G, Ratti S, Fiume R, Yung Follo M, Billi AM, Rusciano I, et al. Phosphoinositide 3 kinase signaling in human stem cells from reprogramming to differentiation: a tale in cytoplasmic and nuclear compartments. International journal of molecular sciences. 2019;20(8):2026.

58. Di Benedetto A, Watkins M, Grimston S, Salazar V, Donsante C, Mbalaviele G, et al. $\mathrm{N}$-cadherin and cadherin 11 modulate postnatal bone growth and osteoblast differentiation by distinct mechanisms. J Cell Sci. 2010;123(15):2640-8.

59. Shen B, Vardy K, Hughes P, Tasdogan A, Zhao Z, Yue R, et al. Integrin alpha11 is an Osteolectin receptor and is required for the maintenance of adult skeletal bone mass. Elife. 2019;8:e42274.

60. Zhang W, Chen E, Chen M, Ye C, Qi Y, Ding Q, et al. IGFBP7 regulates the osteogenic differentiation of bone marrow-derived mesenchymal stem cells via Wnt/ß-catenin signaling pathway. The FASEB Journal. 2018;32(4):2280-91.

61. Malysheva K. Rooij Kd, WGM Löwik C, L Baeten D, Rose-John S. Interleukin 6/Wnt interactions in rheumatoid arthritis: interleukin 6 inhibits Wnt signaling in synovial fibroblasts and osteoblasts. Croatian medical journal. 2016:57(2):89-98.

62. Li X, Zhou Z-y, Zhang Y-y, Yang H-I. IL-6 contributes to the defective osteogenesis of bone marrow stromal cells from the vertebral body of the glucocorticoid-induced osteoporotic mouse. PLoS One. 2016;11(4):e0154677.

63. Langhammer T-S, Roolf C, Krohn S, Kretzschmar C, Huebner R, Rolfs A, et al. PI3K/Akt signaling interacts with Wnt/ $\beta$-Catenin signaling but does not induce an accumulation of $\beta$-catenin in the nucleus of acute lymphoblastic leukemia cell lines. DC: American Society of Hematology Washington; 2013.

64. Han L, Yang Y, Yue X, Huang K, Liu X, Pu P, et al. Inactivation of PI3K A AKT signaling inhibits glioma cell growth through modulation of $\beta$-cateninmediated transcription. Brain research. 2010;1366:9-17.

65. Fang D, Hawke D, Zheng Y, Xia Y, Meisenhelder J, Nika H, et al. Phosphorylation of $\beta$-catenin by AKT promotes $\beta$-catenin transcriptional activity. Journal of Biological Chemistry. 2007;282(15):11221-9.

66. Duan X, Murata Y, Liu Y, Nicolae C, Olsen BR, Berendsen AD. Vegfa regulates perichondrial vascularity and osteoblast differentiation in bone development. Development. 2015;142(11):1984-91.

67. Grosso A, Burger MG, Lunger A, Schaefer DJ, Banfi A, Di Maggio N. It takes two to tango: coupling of angiogenesis and osteogenesis for bone regeneration. Frontiers in bioengineering and biotechnology. 2017:5:68.

68. Maes C, Goossens S, Bartunkova S, Drogat B, Coenegrachts L, Stockmans I, et al. Increased skeletal VEGF enhances $\beta$-catenin activity and results in excessively ossified bones. The EMBO journal. 2010;29(2):424-41.

69. Olsen JJ, Pohl SÖ-G, Deshmukh A, Visweswaran M, Ward NC, Arfuso F, et al. The role of Wnt signalling in angiogenesis. The Clinical Biochemist Reviews. 2017:38(3):131.

70. Ruijtenberg S, van den Heuvel S. Coordinating cell proliferation and differentiation: Antagonism between cell cycle regulators and cell typespecific gene expression. Cell cycle. 2016;15(2):196-212.

71. Jiang $H$, Hong $T$, Wang $T$, Wang $X$, Cao $L, X u X$, et al. Gene expression profiling of human bone marrow mesenchymal stem cells during osteogenic differentiation. Journal of cellular physiology. 2019;234(5): 7070-7.

72. Li L, Zhang C, Chen Jl, Hong Ff, Chen P, Wang Jf. Effects of simulated microgravity on the expression profiles of RNA during osteogenic differentiation of human bone marrow mesenchymal stem cells. Cell proliferation. 2019:52(2):e12539.

73. Davidson G, Shen J, Huang Y-L, Su Y, Karaulanov E, Bartscherer K, et al. Cell cycle control of wnt receptor activation. Developmental cell. 2009;17(6):788-99. 
74. Niehrs C, Acebron SP. Mitotic and mitogenic Wnt signalling. The EMBO journal. 2012;31(12):2705-13.

75. Červenka I, Wolf J, Mašek J, Krejci P, Wilcox WR, Kozubík A, et al. Mitogenactivated protein kinases promote WNT/ $\beta$-catenin signaling via phosphorylation of LRP6. Molecular and cellular biology. 2011;31(1):179-89.

76. Zhang M, Pritchard MR, Middleton FA, Horton JA, Damron TA. Microarray analysis of perichondral and reserve growth plate zones identifies differential gene expressions and signal pathways. Bone. 2008:43(3):511-20.

77. Wodarz A, Nusse R. Mechanisms of Wnt signaling in development. Annual review of cell and developmental biology. 1998;14(1):59-88.

78. Huelsken J, Vogel R, Brinkmann V, Erdmann B, Birchmeier C, Birchmeier W. Requirement for $\beta$-catenin in anterior-posterior axis formation in mice. The Journal of cell biology. 2000;148(3):567-78.

79. Houschyar KS, Tapking C, Borrelli MR, Popp D, Duscher D, Maan ZN, et al. Wnt pathway in bone repair and regeneration-what do we know so far. Frontiers in cell and developmental biology. 2018;6.

80. James AW. Review of signaling pathways governing MSC osteogenic and adipogenic differentiation. Scientifica. 2013;2013.

81. Gaur T, Lengner CJ, Hovhannisyan H, Bhat RA, Bodine PV, Komm BS, et al. Canonical WNT signaling promotes osteogenesis by directly stimulating Runx2 gene expression. Journal of Biological Chemistry. 2005;280(39):33132-40.

82. Tornero-Esteban P, Peralta-Sastre A, Herranz E, Rodríguez-Rodríguez L, Mucientes A, Abásolo L, et al. Altered expression of Wnt signaling pathway components in osteogenesis of mesenchymal stem cells in osteoarthritis patients. PLoS One. 2015;10(9):e0137170.

83. Novak A, Hsu S-C, Leung-Hagesteijn C, Radeva G, Papkoff J, Montesano R, et al. Cell adhesion and the integrin-linked kinase regulate the LEF-1 and $\beta$ catenin signaling pathways. 1998;95(8):4374-9.

84. Ishii T, Furuoka H, Muroi Y, Nishimura MJJoBC. Inactivation of integrin-linked kinase induces aberrant tau phosphorylation via sustained activation of glycogen synthase kinase 3ß in N1E-115 neuroblastoma cells. 2003;278(29): 26970-5.

85. Saidak Z, Le Henaff C, Azzi S, Marty C, Da Nascimento S, Sonnet P, et al. Wnt/ $\beta$-catenin signaling mediates osteoblast differentiation triggered by peptide-induced a5 $\beta 1$ integrin priming in mesenchymal skeletal cells. Journal of Biological Chemistry. 2015;290(11):6903-12.

86. Desbois-Mouthon C, Cadoret A, Blivet-Van Eggelpoel M-J, Bertrand F, Cherqui G, Perret $C$, et al. Insulin and IGF-1 stimulate the $\beta$-catenin pathway through two signalling cascades involving GSK-3 $\beta$ inhibition and Ras activation. Oncogene. 2001;20(2):252.

87. Morali OG, Delmas V, Moore R, Jeanney C, Thiery JP, Larue L. IGF-II induces rapid $\beta$-catenin relocation to the nucleus during epithelium to mesenchyme transition. Oncogene. 2001;20(36):4942.

88. Felber K, Elks PM, Lecca M, Roehl HH. Expression of osterix is regulated by FGF and Wnt/ $\beta$-catenin signalling during osteoblast differentiation. PloS one. 2015;10(12):e0144982.

89. Wang Y, Zhang X, Shao J, Liu H, Liu X. Luo EJSr. Adiponectin regulates BMSC osteogenic differentiation and osteogenesis through the $\mathrm{Wnt} / \beta$ catenin pathway. 2017;7(1):1-13.

90. Alves RD, Eijken M, van de Peppel J, van Leeuwen JP. Calcifying vascular smooth muscle cells and osteoblasts: independent cell types exhibiting extracellular matrix and biomineralization-related mimicries. BMC genomics. 2014;15(1):1-14.

\section{Publisher's Note}

Springer Nature remains neutral with regard to jurisdictional claims in published maps and institutional affiliations.

Ready to submit your research? Choose BMC and benefit from:
- fast, convenient online submission
- thorough peer review by experienced researchers in your field
- rapid publication on acceptance
- support for research data, including large and complex data types
- gold Open Access which fosters wider collaboration and increased citations
- maximum visibility for your research: over 100M website views per year
At BMC, research is always in progress.
Learn more biomedcentral.com/submissions

